\title{
Risk Factor Analysis for Secondary Malignancy in Dexrazoxane-Treated Pediatric Cancer Patients
}

\author{
Hyery Kim, MD, PhD',2 \\ Hyoung Jin Kang, MD, PhD' \\ Kyung Duk Park, MD, PhD' \\ Kyung-Nam Koh, MD, PhD² \\ Ho Joon Im, MD, PhD² \\ Jong Jin Seo, MD, PhD² \\ Jae Wook Lee, MD, $\mathrm{PhD}^{3}$ \\ Nack-Gyun Chung, MD, $\mathrm{PhD}^{3}$ \\ Bin Cho, MD, PhD ${ }^{3}$ \\ Hack Ki Kim, MD, $\mathrm{PhD}^{3}$ \\ Jae Min Lee, MD, $\mathrm{PhD}^{4}$ \\ Jeong Ok Hah, MD, PhD 4,5 \\ Jun Ah Lee, MD, PhD ${ }^{6}$ \\ Young Ho Lee, MD, $\mathrm{PhD}^{7}$ \\ Sang Kyu Park, MD, PhD ${ }^{8}$ \\ Hee Jo Baek, MD, PhD ${ }^{9}$ \\ Hoon Kook, MD, PhD ${ }^{9}$ \\ Ji Yoon Kim, MD, PhD 10,11 \\ Heung Sik Kim, MD, PhD ${ }^{10}$ \\ Hwang Min Kim, MD, PhD ${ }^{12}$ \\ Hee Won Chueh, MD, PhD ${ }^{13}$ \\ Meerim Park, MD, PhD ${ }^{14}$ \\ Hoi Soo Yoon, MD, PhD ${ }^{15}$ \\ Mee Jeong Lee, MD, PhD ${ }^{16}$ \\ Hyoung Soo Choi, MD, PhD ${ }^{17}$ \\ Hyo Seop Ahn, MD, PhD 1,17 \\ Yoshifumi Kawano, MD, $\mathrm{PhD}^{18}$ \\ Ji Won Park, MS ${ }^{19}$ \\ Seokyung Hahn, $\mathrm{PhD}^{19}$ \\ Hee Young Shin, MD, PhD'
}

*A list author's affiliations appears at the end of the paper.

Correspondence: Hee Young Shin, MD, PhD

Division of Hematology/ Oncology,

Department of Pediatrics, Cancer Research

Institute, Seoul National University College

of Medicine, 103 Daehak-ro, Jongno-gu,

Seoul 03080, Korea

Tel: 82-2-2072-2917

Fax: 82-2-743-3455

E-mail: hyshin@snu.ac.kr

Received September 26, 2017

Accepted May 11, 2018

Published Online May 14, 2018

\section{Purpose}

Dexrazoxane has been used as an effective cardioprotector against anthracycline cardiotoxicity. This study intended to analyze cardioprotective efficacy and secondary malignancy development, and elucidate risk factors for secondary malignancies in dexrazoxane-treated pediatric patients.

\section{Materials and Methods}

Data was collected from 15 hospitals in Korea. Patients who received any anthracyclines, and completed treatment without stem cell transplantation were included. For efficacy evaluation, the incidence of cardiac events and cardiac event-free survival rates were compared. Data about risk factors of secondary malignancies were collected.

\section{Results}

Data of total 1,453 cases were analyzed; dexrazoxane with every anthracyclines group (D group, 1,035 patients) and no dexrazoxane group (non-D group, 418 patients). Incidence of the reported cardiac events was not statistically different between two groups; however, the cardiac event-free survival rate of patients with more than $400 \mathrm{mg} / \mathrm{m}^{2}$ of anthracyclines was significantly higher in D group $(91.2 \%$ vs. $80.1 \%, p=0.04)$. The 6-year cumulative incidence of secondary malignancy was not different between both groups after considering follow-up duration difference (non-D, 0.52\% $\pm 0.37 \%$;, $0.60 \% \pm 0.28 \% ; p=0.55$ ). The most influential risk factor for secondary malignancy was the duration of anthracycline administration according to multivariate analysis.

\section{Conclusion}

Dexrazoxane had an efficacy in lowering cardiac event-free survival rates in patients with higher cumulative anthracyclines. As a result of multivariate analysis for assessing risk factors of secondary malignancy, the occurrence of secondary malignancy was not related to dexrazoxane administration. 


\section{Introduction}

Anthracycline plays an important role in chemotherapy of various cancer types, including breast cancer, leukemia, and sarcoma so that about $50 \%$ of pediatric cancer patients are receiving anthracycline based chemotherapy [1]. However, cardiotoxicity is well known side effect of anthracycline, and leaves permanent cardiac damage in certain patients. The likelihood of anthracycline-related cardiotoxicity is reported to be up to $57 \%$, and mortality rate due to these heart problems is reported as 8.2 times higher than that of the normal people [2]. In particular, the risk of late onset cardiotoxicity is reported to be higher in patients younger at the time of anthracycline administration, and received higher total cumulative anthracycline dose.

Because it is important to prevent late anthracycline induced cardiac event due to irreversibility, various attempts to prevent cardiac toxicity of anthracyclines were tried, such as changing administration methods or co-infusion of candidate cardio-protective agents. However, those methods were not effective in preventing cardiac toxicity only except dexrazoxane (Cardioxane) [1].

Dexrazoxane has been widely used to prevent cardiac toxicity after anthracycline administration including pediatric cancer patients, and showed clinically significant cardio-protective effects $[3,4]$. However, in a previous prospective study of Hodgkin's disease, there was a claim that dexrazoxane might have increased the incidence of myelodysplastic syndrome (MDS) and secondary cancers [5].

However, on the contrary to the report with Hodgkin's disease, following studies with various diseases and large numbers of patients showed that there was no difference in the occurrence of secondary malignancies (SMN) between patients administered dexrazoxane and the others [6-8]. In addition, there was no previous report about risk factor analysis including multivariate factors influencing the development of SMN. It is needed to verify whether SMN occurs in patients who received dexrazoxane, and whether other risk factors are associated with SMN. This study intended to analyze cardioprotective efficacy and SMN development related to dexrazoxane, and find out risk factors for SMN in Korean patients who received dexrazoxane.

\section{Materials and Methods}

\section{Study population}

This nationwide study was conducted in 15 institutions in Korea. The subjects for this study were selected from all pediatric cancer patients whose treatment was ended at the time of study initiation (November 2012). Among those, patients who had received anthracycline during their chemotherapy and did not receive hematopoietic stem cell transplantation were finally selected for the analysis. The patients were divided into two groups: (1) patients who used dexrazoxane with anthracyclines, and (2) patients who did not use the dexrazoxane. For the comparison with control group, patients who had received dexrazoxane with every dose of anthracyclines during their treatment regardless of the preceding risk factors for cardiotoxicity were included, and those who were administered dexrazoxane intermittently or only during selected schedules were excluded.

\section{Data collection}

Various clinical and laboratory data were collected, which included patients' demographics, diagnosis, chemotherapy history, dexrazoxane related data (total dose, date of first and last administration), any cardiac event, survival status and other late effects at the last follow up. Data reported to be risk factors of SMN were also collected including any congenital condition or hereditary syndrome, granulocyte colony-stimulating factor (G-CSF) administration and the details of radiation therapy. Irradiation on the thoraxinvolved fields (thorax, lung, craniospinal, mediastinum, etc.) might influence late cardiac function, thus radiation in those locations was considered as an independent factor for the analysis of late cardiac function. Cumulative doses of various chemotherapeutic agents including anthracyclines (daunorubicin, doxorubicin, idarubicin, epirubicin, and mitoxantrone), etoposide and cyclophosphamide were calculated. All anthracycline doses were converted to doxorubicin equivalent doses with the formula using doxorubicin equivalent dose conversion [9].

All the information was obtained using the electronic case report form developed by the Medical Research Collaborating Center (MRCC) in Seoul National University of Medicine, and was inputted by a designated researcher at each hospital. Data-management experts at MRCC performed quality-control checks to ensure accuracy of the recorded data, and audits were conducted thoroughly. 


\section{Statistical analysis}

Baseline and demographic characteristics were summarized by standard descriptive statistics. Cumulative doses of chemotherapeutic agents and data related to dexrazoxane were summarized by descriptive statistics. For continuous data, basic statistical values were obtained, and T-test for parametric data and Wilcoxon rank sum test for nonparametric data were conducted. In case of categorical data, the frequencies and percentages were obtained, and Pearson's chi-square test or Fisher exact test were conducted for comparison between groups.

Events were defined as any relapse, death, or SMN. Cardiac events were defined as any cardiac abnormalities detected during and after the administration of anthracyclines including sudden cardiac death, and early cardiac toxicity. We defined early cardiotoxicity as which developed during treatment or first year after treatment completion, whereas late cardiotoxicity as which developed at least one year after therapy in cancer survivors $[10,11]$. Probabilities of event-free survival (EFS) and overall survival (OS) were estimated by Kaplan-Meier method, and survival differences were analyzed with log-rank test. Cox proportional hazard regression model was made after being adjusted for baseline and other factors which might influence on the outcome variables. Cardiac EFS was separately calculated for four groups which were divided according to each quartile values of the cumulative doses of anthracyclines and dexrazoxane.

The incidence of SMN and death in the two groups and unadjusted odds ratios were estimated with $95 \%$ confidence intervals. Considering the competing risks for death or relapse, cumulative incidence curves were calculated, and Fine and Gray models were used for the analysis of the difference between two groups. In addition, competing risk analysis with Fine and Gray model was conducted for the evaluation of risk factors for SMN. The correction factors for competing risk analysis were age, sex, diagnosis, G-CSF administration, hereditary or genetic syndrome, cumulative doses of chemotherapeutics, irradiation history, duration of anthracycline administration, duration between first anthracycline and last echocardiography, and total duration of chemotherapy.

All the statistical analysis was performed by biostatistics specialists at the MRCC in Seoul National University of Medicine, and SAS ver. 9.4 (SAS Institute Inc., Cary, NC) was used as a statistics program.

\section{Ethical statement}

This study has been approved by the Institutional Review Board of Seoul National University Hospital (H-1212-107453), and each participating hospital with a waiver of informed consent.

\section{Results}

\section{Characteristics of the patients}

Data of total 1,788 patients from 15 institutions were collected, and data of total 1,453 patients were analyzed for the study (S1 Fig., S2 Table). The date of initial diagnosis of all patients were ranged from August 1996 to September 2012. Dexrazoxane group (D group) included 1,035 patients, and non-dexrazoxane group (non-D group) included 418 patients. Median age at diagnosis was 5 years in non-D group, and 6 years in D group (Table 1). Age and sex proportions were not statistically different between two groups, but follow-up duration was longer in non-D group, as median 124.3 months comparing with 59.4 months in $\mathrm{D}$ group $(\mathrm{p}<0.01)$.

Diagnosis of patients was various as shown in Table 1. In D group, there were acute lymphoblastic leukemia (26.5\%), non-Hodgkin's lymphoma (16.0\%), and osteosarcoma (12.2\%) in the order of proportion, and acute lymphoblastic leukemia $(42.6 \%)$, non-Hodgkin's lymphoma $(18.7 \%)$ and neuroblastoma $(6.5 \%)$ in non-D group. The distribution of diagnosis was statistically different between two groups $(\mathrm{p}<0.01)$.

We collected data of hereditary syndrome or genetic disease in all subjects (Table 1). There were 12 patients who were diagnosed with hereditary syndrome or genetic diseases; two $(0.5 \%)$ patients in non-D group and $10(1.0 \%)$ patients in D group, respectively.

\section{Chemotherapy and dexrazoxane}

Cumulative doses of chemotherapeutic agents are shown in Table 2. The median cumulative doses of etoposide were $1,830 \mathrm{mg} / \mathrm{m}^{2}$ in non-D group and $1.82 \mathrm{~g} / \mathrm{m}^{2}$ in $\mathrm{D}$ group $(\mathrm{p}=0.52)$. Cumulative doses of cyclophosphamide were significantly higher in the $\mathrm{D}$ group, as median cumulative doses were $4,030 \mathrm{mg} / \mathrm{m}^{2}$ in D group and $2,570 \mathrm{mg} / \mathrm{m}^{2}$ in non-D group $(\mathrm{p}<0.01)$. The data about anthracyclines doses was collected in 1,443 patients, and the dose of total cumulative anthracyclines was significantly higher in D group, as 210 $\mathrm{mg} / \mathrm{m}^{2}$ in $\mathrm{D}$ group and $150 \mathrm{mg} / \mathrm{m}^{2}$ in non-D group, respectively $(p<0.01)$. Among anthracyclines, cumulative doses of doxorubicin and idarubicin were significantly higher in the D group.

The median duration of entire chemotherapy was 652 days in non-D group and 366 days in $\mathrm{D}$ group, and total duration of chemotherapy was significantly longer in non-D group $(p<0.01)$ (Table 2), and the median total duration of anthra- 
Table 1. Clinical characteristics of all patients

\begin{tabular}{|c|c|c|c|}
\hline Characteristic & $\begin{array}{l}\text { Non-dexrazoxane } \\
\qquad(\mathrm{n}=418)\end{array}$ & $\begin{array}{c}\text { Dexrazoxane } \\
(n=1,035)\end{array}$ & p-value \\
\hline Age at diagnosis (yr) & $5(0-26)$ & $6(0-21)$ & 0.10 \\
\hline \multicolumn{4}{|l|}{ Sex } \\
\hline Female & $174(41.6)$ & $411(39.7)$ & 0.50 \\
\hline Male & $244(58.4)$ & $624(60.3)$ & \\
\hline Follow-up duration (mo) & $124.3(0-209.2)$ & $59.4(0-182.1)$ & $<0.01$ \\
\hline \multicolumn{4}{|l|}{ Diagnosis } \\
\hline Acute lymphoblastic leukemia & $178(42.6)$ & $274(26.5)$ & $<0.01$ \\
\hline Acute myeloid leukemia & $23(5.5)$ & $61(5.9)$ & \\
\hline Acute biphenotypic leukemia & $9(2.2)$ & $8(0.8)$ & \\
\hline Ewing/Primitive neuroectodermal tumor & $7(1.7)$ & $44(4.3)$ & \\
\hline Extracranial germ cell tumor & $5(1.2)$ & $26(2.5)$ & \\
\hline Hepatic tumor & $18(4.3)$ & $39(3.8)$ & \\
\hline Hodgkin lymphoma & $12(2.9)$ & $34(3.3)$ & \\
\hline Leukemia other & $2(0.5)$ & $4(0.4)$ & \\
\hline Neuroblastoma & $27(6.5)$ & $78(7.5)$ & \\
\hline Non-Hodgkin's lymphoma & $78(18.7)$ & $166(16.0)$ & \\
\hline Non-rhabdomyosarcoma soft-tissue sarcomas & $8(1.9)$ & $34(3.3)$ & \\
\hline Osteosarcoma & $20(4.8)$ & $126(12.2)$ & \\
\hline Other solid tumor & $5(1.2)$ & $14(1.4)$ & \\
\hline Renal tumor & $17(4.1)$ & $30(2.9)$ & \\
\hline Retinoblastoma & $5(1.2)$ & $75(7.2)$ & \\
\hline Rhabdomyosarcoma & $4(1.0)$ & $22(2.1)$ & \\
\hline \multicolumn{4}{|l|}{ Hereditary syndrome or genetic disease } \\
\hline No & $416(99.5)$ & $1,025(99.0)$ & 0.53 \\
\hline Yes & $2(0.5)$ & $10(1.0)$ & \\
\hline $13 q$ deletion syndrome & 0 & $2(20.0)$ & \\
\hline Beckwith-Wiedemann syndrome & 0 & $1(10.0)$ & \\
\hline Marfans syndrome & $1(50.0)$ & 0 & \\
\hline Neurofibromatosis & 0 & $2(20.0)$ & \\
\hline Noonan syndrome & 0 & $2(20.0)$ & \\
\hline Rothmund Thompson syndrome & 0 & $1(10.0)$ & \\
\hline Tuberous sclerosis & $1(50.0)$ & 0 & \\
\hline WAGR 11P13 deletion syndrome & 0 & $1(10.0)$ & \\
\hline Williams syndrome & 0 & $1(10.0)$ & \\
\hline
\end{tabular}

Values are presented as median (range) or number (\%).

cycline administration was 161 days in non-D group and 168 days in $\mathrm{D}$ group ( $\mathrm{p}=0.90$ ). The median age of first anthracycline administration was 5 years in non-D group and 6 years in $\mathrm{D}$ group, respectively ( $\mathrm{p}=0.73$ ). The median dose of dexrazoxane was $2,390 \mathrm{mg} / \mathrm{m}^{2}$, and the median total duration of dexrazoxane administration was 167 days (range, 1 to 2,974 days).

Routine G-CSF administration during neutropenia was more frequent in $\mathrm{D}$ group $(86.0 \%)$ than in non-D group $(71.2 \%)(\mathrm{p}<0.01)$, and more patients in non-D group $(32.1 \%)$ received radiotherapy $(\mathrm{p}<0.01)$ (Table 2$)$. The total dose of radiation was significantly higher in $\mathrm{D}$ group $(\mathrm{p}=0.01)$; however, radiation fields were various and the number of patients who received radiation therapy on thorax-related fields was not significantly different between two groups $(\mathrm{p}=0.48)$.

\section{Efficacy of dexrazoxane}

Cardiac events in 1,452 patients until the time of data collection were collected. A total of 75 cardiac events occurred, and there were 16 cases (3.8\%) in non-D group, and 59 cases 
Table 2. Cumulative doses of chemotherapeutics and details of treatment

\begin{tabular}{|c|c|c|c|c|c|}
\hline & \multicolumn{2}{|c|}{ Non-dexrazoxane $(\mathrm{n}=418)$} & \multicolumn{2}{|c|}{ Dexrazoxane $(n=1,035)$} & \multirow{2}{*}{ p-value } \\
\hline & No. $(\%)$ & Median (range) & No. $(\%)$ & Median (range) & \\
\hline \multicolumn{6}{|l|}{ Cumulative doses of chemotherapeutics $\left(\mathrm{mg} / \mathrm{m}^{2}\right)$} \\
\hline Etoposide & 122 & $1,830(120-6,340)$ & 447 & $1,820(40-15,430)$ & 0.52 \\
\hline Cyclophosphmide & 297 & $2,570(310-35,370)$ & 718 & $4,030(4-74,110)$ & $<0.01$ \\
\hline Total anthracyclines ${ }^{\text {a) }}$ & $411^{\mathrm{b})}$ & $150(15.4-665.1)$ & $1,032^{\mathrm{c})}$ & $210(17-753.9)$ & $<0.01$ \\
\hline Daunomycin (daunorubicin) & 164 & $75(18-347)$ & 304 & $80(18.4-392)$ & 0.20 \\
\hline Doxorubicin (adriamycin) & 364 & $120(15-691)$ & 909 & $180(17.3-665)$ & $<0.01$ \\
\hline Idarubicin & 27 & $50(6-113)$ & 80 & $60(10.7-248)$ & 0.03 \\
\hline Epirubicin & 1 & 208 & 13 & $100(31.1-1,091)$ & 1.00 \\
\hline Mitoxantrone & 15 & $40(16-100)$ & 35 & $40(12-55)$ & 0.73 \\
\hline Total duration of chemotherapy (day) $)^{\text {d) }}$ & - & $652(0-2,655)$ & - & $366(1-3,444)$ & $<0.01$ \\
\hline Total duration of anthracycline (day) $)^{\mathrm{d})}$ & - & $161(0-1,889)$ & - & $168(0-2,974)$ & 0.90 \\
\hline Age at first anthracycline (yr) & - & $5(0-26)$ & - & $6(0-21)$ & 0.73 \\
\hline Total dose of dexrazoxane $\left(\mathrm{mg} / \mathrm{m}^{2}\right)$ & - & - & - & $2,390(125-36,989)$ & $<0.01$ \\
\hline Total duration of dexrazoxane (day) ${ }^{\mathrm{d})}$ & - & - & - & $167(1-2,974)$ & $<0.01$ \\
\hline G-CSF administration during neutropenia & $297(71.2)$ & - & $890(86.0)$ & - & $<0.01$ \\
\hline Radiotherapy & $134(32.1)$ & - & $201(19.4)$ & - & $<0.01$ \\
\hline Radiotherapy on thorax & $9(2.2)$ & - & $29(2.8)$ & - & 0.48 \\
\hline Radiation dose (Gy) & - & $18(2-63)$ & - & $22.5(0.45-147)$ & 0.01 \\
\hline
\end{tabular}

G-CSF, granulocyte colony-stimulating factor. ${ }^{a}$ Cumulative dose of total anthracyclines $\left(\mathrm{mg} / \mathrm{m}^{2}\right)=$ daunorubicin $\left(\mathrm{mg} / \mathrm{m}^{2}\right) \times$ $0.833+$ doxorubicin $\left(\mathrm{mg} / \mathrm{m}^{2}\right)+$ idarubicin $\left(\mathrm{mg} / \mathrm{m}^{2}\right) \times 5+$ epirubicin $\left(\mathrm{mg} / \mathrm{m}^{2}\right) \times 0.67+$ mitoxantrone $\left.\left(\mathrm{mg} / \mathrm{m}^{2}\right) \times 4,{ }^{b}\right)$ Dose data of 7 patients were not available, ${ }^{\mathrm{c}}$ Dose data of 3 patients were not available, ${ }^{\mathrm{d}}$ Total duration of the drug=date of last administration-date of first administration.

A
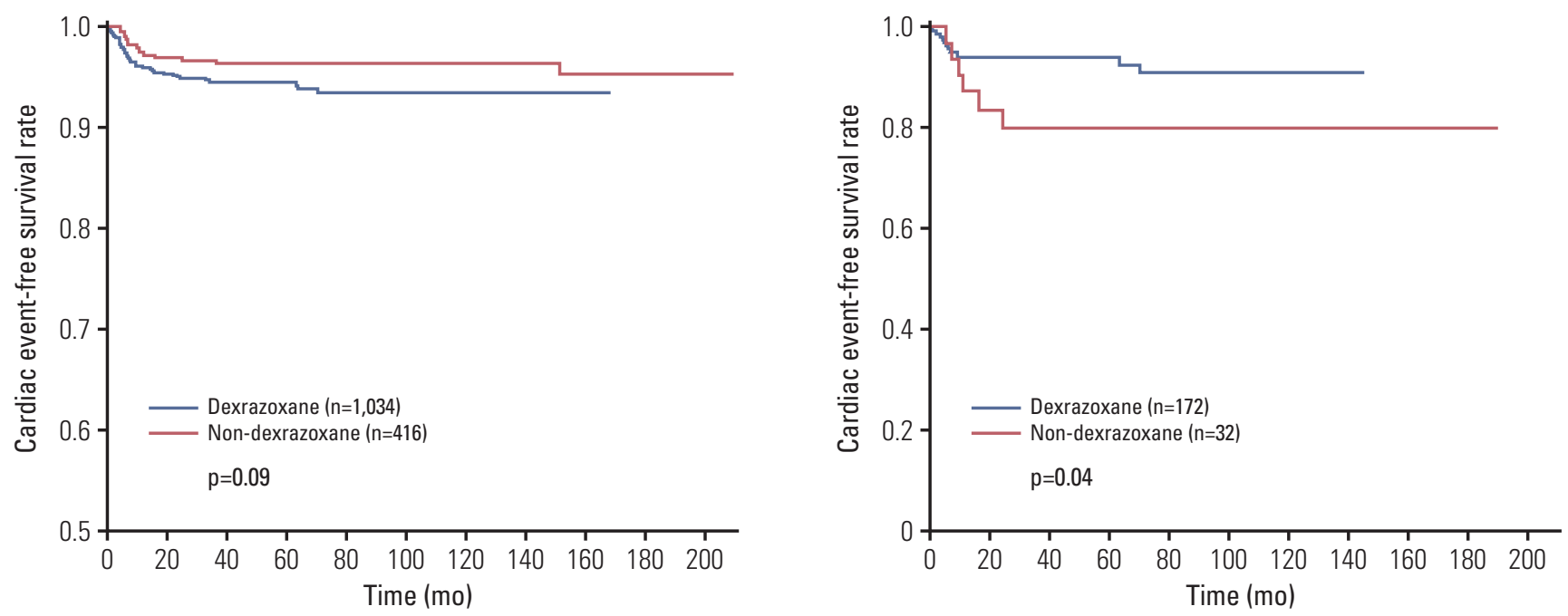

Fig. 1. Cardiac event-free survival (EFS) of all patients (A), patients who received more than $400 \mathrm{mg} / \mathrm{m}^{2}$ of total cumulative anthracyclines (B). (A) The cardiac EFS rate was $95.4 \%$ in non-dexrazoxane group (non-D group) ( $n=416$ ), and $93.4 \%$ in dexrazoxane group (D group) $(n=1,034)(p=0.09)$, (B) but there was significant difference in cardiac EFS between non-D group $(\mathrm{n}=32)$ and $\mathrm{D}$ group of patients $(\mathrm{n}=172)$ who received more than $400 \mathrm{mg} / \mathrm{m}^{2}$, as $80.1 \%$ and $91.2 \%(\mathrm{p}=0.04)$. 


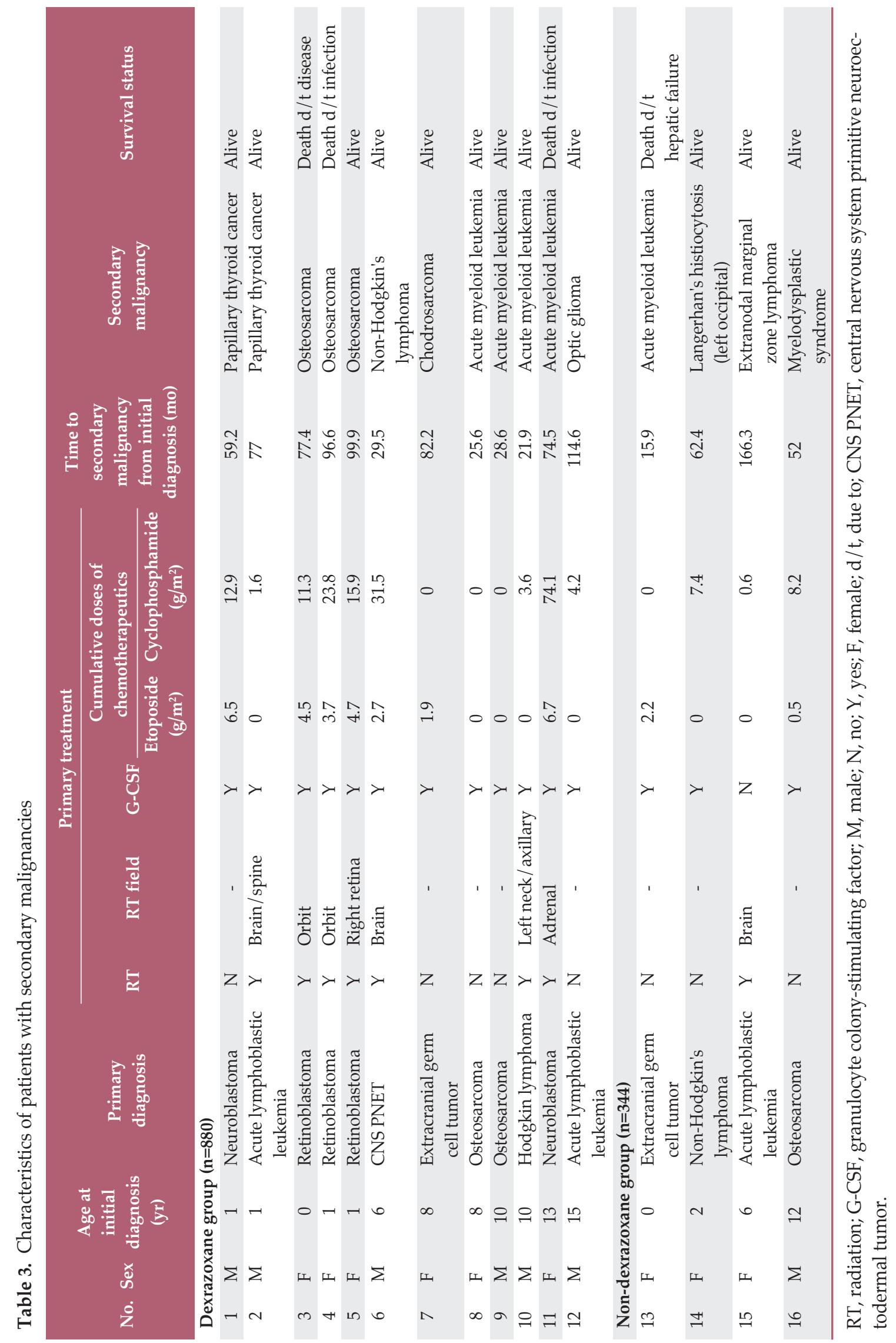



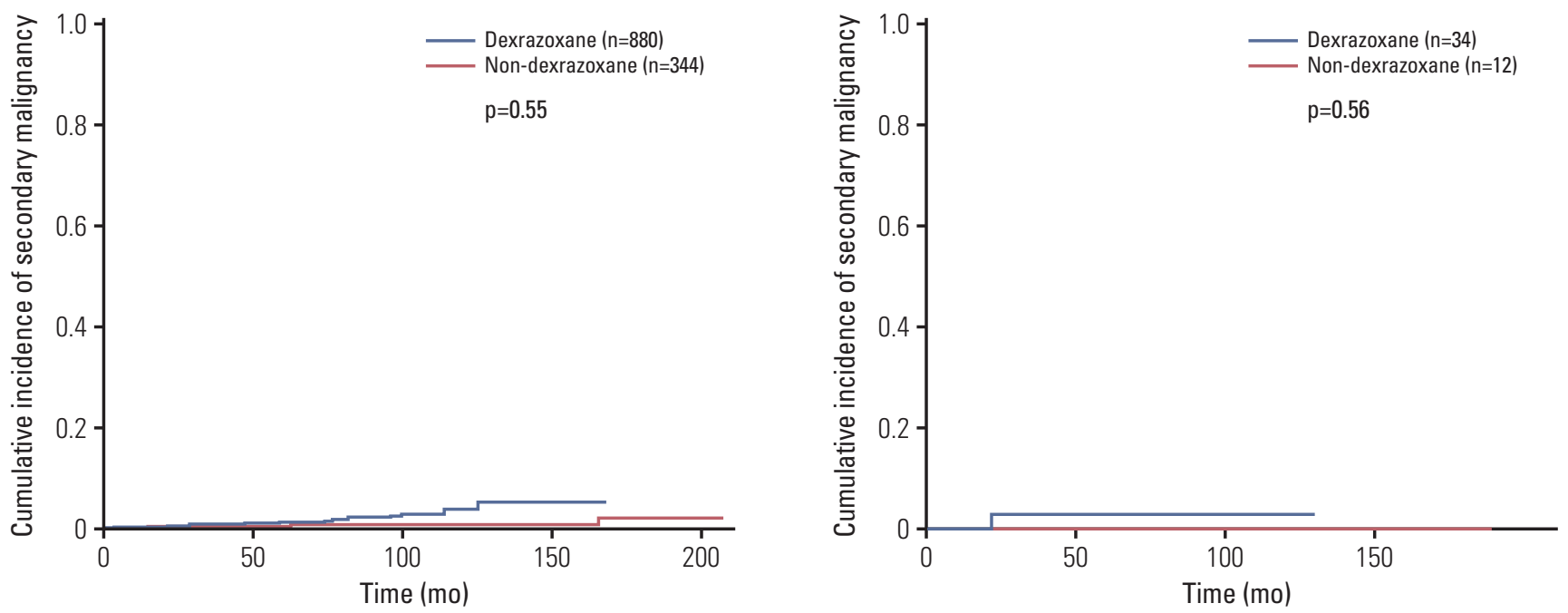

Fig. 2. The 6-year cumulative incidence rate of secondary malignancy in all patients $(n=1,224)(A)$, patients with Hodgkin disease ( $\mathrm{n}=46$ ) (B). (A) The 6-year cumulative incidence rate of secondary malignancies (SMN) in all patients was $0.67 \pm 0.24 \%$. The rates of SMN were $0.52 \% \pm 0.37 \%$ in non-dexrazoxane group (non-D group) ( $\mathrm{n}=880$ ) and $0.60 \% \pm 0.28 \%$ in dexrazoxane group (D group) $(\mathrm{n}=344)(\mathrm{p}=0.55)$. (B) When patients with Hodgkin lymphoma were analyzed, the rates of SMN was $0 \%$ in non-D group $(n=12)$ and $3.1 \% \pm 2.9 \%$ in D group $(n=34)(p=0.56)$.

$(5.7 \%)$ in D group ( $\mathrm{p}=0.24)$ (S3 Table). Among all cardiac events, asymptomatic ventricular dysfunction in echocardiography was the most common cardiac manifestation (3 cases in non-D group, 26 cases in $\mathrm{D}$ group). According to the time of cardiotoxicity, early cardiotoxicity occurred in 13 cases of non-D group, and 52 cases of $\mathrm{D}$ group.

The cardiac EFS rate was $95.4 \%$ in non-D group, and $93.4 \%$ in D group. There was no significant difference in EFS after considering the corrections factors $(\mathrm{p}=0.09)$ (Fig. 1A). According to the total anthracyclines cumulative doses, there were 204 patients who received more than $400 \mathrm{mg} / \mathrm{m}^{2}$ of anthracyclines (32 patients in non-D group, 172 patients in D group). There were 6 cardiac events in non-D group, and 12 events in D group (S4 Table). When analyzing the cardiac EFS rates according to the total cumulative anthracycline doses, there was significant difference between non-D group and D group of patients who received more than $400 \mathrm{mg} / \mathrm{m}^{2}$, as $80.1 \%$ and $91.2 \%$ ( $\mathrm{p}=0.04$ ) (Fig. 1B).

\section{Secondary malignancy and survival}

Data of SMN was available in 1,224 patients (non-D group 344 patients and D group 880 patients), and there were 4 and 12 patients who developed SMN or MDS in non-D group and D group, respectively (Table 3 ). The median latency from initial diagnosis to SMN was 5.7 years (range, 1.3 to 13.9 years). The most common SMN was acute myeloid leukemia
(AML), as total five patients developed AML, and osteosarcoma was developed in three patients. The follow up duration of both groups were significantly different, so the cumulative incidence of both groups were compared at the same time points. The 6-year cumulative incidence rate of SMN in all patients was $0.67 \pm 0.24 \%$. The rates of SMN were $0.52 \% \pm$ $0.37 \%$ in non-D group and $0.60 \% \pm 0.28 \%$ in $\mathrm{D}$ group, respectively (Fig. 2A), and the cumulative incidence rate after considering correction factors was not statistically different between two groups $(\mathrm{p}=0.55)$. When patients with Hodgkin lymphoma were analyzed (non-D group 12 patients, and D group 34 patients), the 6-year cumulative incidence rate of SMN was $0 \%$ in non-D group and $3.1 \% \pm 2.9 \%$ in D group, and the difference between two groups was not significant $(\mathrm{p}=0.56)$ (Fig. 2B).

The OS in D group was $87.4 \%$, and that in non-D group was $86.3 \%$ (Fig. 3A). The EFS was $80.3 \%$ in D group and $80.4 \%$ in non-D group (Fig. 3B). The survival rates were not significantly different between two groups (OS, $\mathrm{p}=0.06$; $\mathrm{EFS}$, $\mathrm{p}=0.64$ ).

\section{Risk factor for secondary malignancy}

Risk factors affecting the occurrence of SMN after considering relapse and death as competing risks were analyzed with Fine and Gray model, and the results are shown in Table 4. According to the univariate analysis with significant 
A
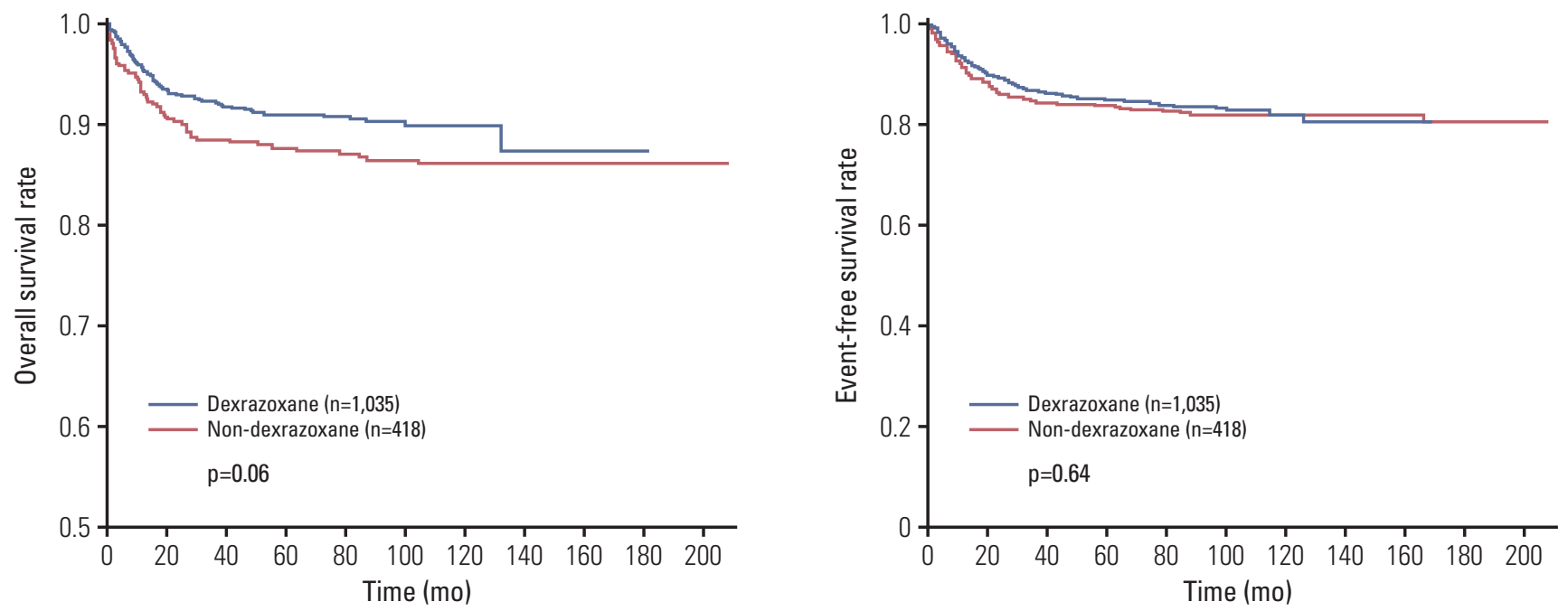

Fig. 3. Survival rates of all patients. (A) Overall survival rate of 1,035 patients in dexrazoxane group (D group) was $87.4 \%$, and that of 418 patients in non-dexrazoxane group (non-D group) was $86.3 \%$ ( $\mathrm{p}=0.06$ ). (B) Event-free survival rate was $80.3 \%$ in $\mathrm{D}$ group $(\mathrm{n}=1,035)$ and $80.4 \%$ in non-D group $(\mathrm{n}=418)(\mathrm{p}=0.64)$.

Table 4. Analysis of the risk factors for secondary malignancy

\begin{tabular}{|c|c|c|c|c|}
\hline \multirow{2}{*}{ Factor } & \multicolumn{2}{|c|}{ Univariate analysis } & \multicolumn{2}{|c|}{ Multivariate analysis } \\
\hline & Unadjusted HR (95\% CI) & p-value & Adjusted HR (95\% CI) & p-value \\
\hline Dexrazoxane group vs. non-dexrazoxane group & $3.46(0.92-13.07)$ & 0.07 & - & - \\
\hline Age $(y r)$ & $0.97(0.85-1.09)$ & 0.55 & - & - \\
\hline Sex (female vs. male) & $2.30(0.82-6.47)$ & 0.11 & - & - \\
\hline Syndrome or genetic disease (yes vs. no) & $3.14(0.03-23.39)$ & 0.50 & - & - \\
\hline G-CSF (yes vs. no) & $5.05(1.03-91.25)$ & 0.09 & - & - \\
\hline Previous radiation (yes vs. no) & $3.12(1.15-8.45)$ & 0.03 & - & - \\
\hline Cumulative dose of etoposide $\left(\mathrm{mg} / \mathrm{m}^{2}\right)$ & $1.31(1.16-1.47)$ & $<0.01$ & - & - \\
\hline Cumulative dose of cyclophosphamide $\left(\mathrm{mg} / \mathrm{m}^{2}\right)$ & $1.08(1.06-1.10)$ & $<0.01$ & - & - \\
\hline Cumulative dose of total anthracyclines $\left(\mathrm{mg} / \mathrm{m}^{2}\right)$ & $2.88(1.82-4.58)$ & $<0.01$ & - & - \\
\hline Total duration of anthracycline (mo) & $1.06(1.05-1.07)$ & $<0.01$ & $1.05(1.03-1.06)$ & $<0.01$ \\
\hline Time after last anthracycline (mo) & $0.98(0.97-0.99)$ & $<0.01$ & $0.99(0.98-0.99)$ & $<0.01$ \\
\hline Total duration of chemotherapy (mo) & $1.05(1.04-1.07)$ & $<0.01$ & - & - \\
\hline
\end{tabular}

$\mathrm{HR}$, hazard ratio; $\mathrm{CI}$, cumulative incidence.

level of $5 \%$, irradiation history, higher cumulative doses of etoposide, cyclophosphamide and anthracyclines, longer duration of anthracycline, shorter time after last anthracycline, and longer duration of chemotherapy were related with statistically significant higher hazard ratio of the occurrence of SMN. The result of multivariate analysis showed that the incidence of SMN was not increased due to dexrazoxane, but increased approximately 1.05 times in response to every 1-month prolongation of anthracycline administration $(\mathrm{p}<0.01)$ (Table 4). Whereas, the incidence of SMN was reduced by about 0.99 times as every month after last anthracycline went by $(\mathrm{p}<0.01)$. 


\section{Discussion}

In this study, multivariate analysis for assessing risk factors of SMN in pediatric cancer patients who received dexrazoxane was conducted, and there was no increased incidence or risk of SMN in those patients.

The mechanism of anthracycline's early cardiotoxicity is known to be related to free radical injury, contributing to the formation of reactive oxygen species and leading to the apoptosis of cardiomyocyte and intracellular damage [12]. On the other hand, the mechanism of delayed cardiotoxicity in the long-term survivors is multifactorial including myocardial mitochondria related apoptosis which results in metabolic remodeling of heart [13]. However, delayed cardiotoxicity presents as overt clinical manifestations such as heart failure only in extreme cases, and only slowly progressing ventricular abnormalities are detected in many cases [13].

For assessing cardiotoxicity in pediatric cancer patients, cardiac event has been counted as one of the markers for cardiotoxicity [3,14]. A cumulative dose of anthracycline has been known as the most significant risk factor for cardiac dysfunction. The incidence of heart failure was approximately $3.0 \%$ in patients receiving a cumulative dose of doxorubicin of $400 \mathrm{mg} / \mathrm{m}^{2}, 7.5 \%$ at doses of $550 \mathrm{mg} / \mathrm{m}^{2}$, and $18.0 \%$ at doses of $700 \mathrm{mg} / \mathrm{m}^{2}$ [15]. In our study, although there was no significant difference in EFS between two groups and the median follow up duration was significantly longer in non-D group, patients who received more than $400 \mathrm{mg} / \mathrm{m}^{2}$ of cumulative anthracyclines and dexrazoxane showed a slightly higher cardiac EFS than the other patients without dexrazoxane. These results might be related to the relatively low incidence of clinical cardiologic events in patients with less than $400 \mathrm{mg} / \mathrm{m}^{2}$ of anthracycline, thus the protective effect would be apparent only in patients receiving higher amounts of anthracyclines. Longer follow-up would be needed considering late cardiotoxicity especially in patients with short treatment off period.

Despite of the cardioprotective effects of dexrazoxane, dexrazoxane was reported to be related with increased risk of SMN. Tebbi et al. [5] reported the results of the Pediatric Oncology Group study with Hodgkin lymphoma, in which patients were randomly assigned to receive dexrazoxane to evaluate its cardiopulmonary protective effect. The authors found an increased incidence of SMN after a median followup time of 4.8 years, especially AML or MDS, in the dexrazoxane-received patients.

However, many refutations against the Tebbi's report have been followed. Since in that article, risk factors of SMN such as cumulative doses of etoposide, cyclophosphamide, and doxorubicin or G-CSF administration were not considered as confounding factors in the analysis. Also, many studies in other patient groups have not shown the same results [6-8, 13].

In our study, multiple risk factors known to affect SMN development were collected. In the multivariate analysis, longer duration of anthracycline, and shorter time after last anthracycline were related with statistically significant higher hazard ratio of the occurrence of SMN. There was no significant risk factor related to AML or MDS, specifically. There was no data related to the association of SMN and duration of certain chemotherapeutic agents. Considering the final adjusted hazard ratios in our study were relatively low as 1.05 and 0.99 , our final multivariate factors could not be the most powerful risk factors for SMN. Further analysis could elucidate more influential risks factors for SMN in these patients group.

Risk factors for SMN are known to be multifactorial. Chemotherapy agents known to be carcinogenic are the alkylating agents, topoisomerase II agents, and anthracyclines [16]. In addition, the cumulative dose, the schedule of chemotherapy administration, and the use of multiple drugs all significantly impact the risk of developing SMN [16].

In our study, patient No. 2 in Table 3 developed papillary thyroid cancer within radiation fields. Although there were cases where the irradiated fields and location of SMN were not related, radiation itself has been reported as one of the risk factors for SMN in childhood cancer patients [17-19]. Secondary osteosarcoma was developed in three retinoblastoma patients. The risk of secondary bone tumors in retinoblastoma survivors is higher in patients with bilateral disease or germline mutations in RB gene. In addition, radiation therapy could also increase the risk of secondary osteosarcoma in retinoblastoma patients [20].

When patients with Hodgkin disease were analyzed separately, dexrazoxane was not a significant risk factor for SMN and the cumulative incidence of SMN was not significantly different regardless of dexrazoxane. In the study of the Childhood, Adolescent and Young Adult Cancer Survivors Research Program of British Columbia which analyzed long term results of 442 Hodgkin lymphoma survivors, radiation therapy (hazard ratio, 2.7), and female sex (hazard ratio, 1.8) were significant risk factors for SMN in multivariate analysis [21]. To analyze risk factors for SMN in Hodgkin disease, many factors other than dexrazoxane should be considered.

This study has several limitations. First, there would be possibility of selection bias due to retrospective design although data management and analysis were conducted by medical statistics specialists. Second, the follow up duration was variable, and there were patients with insufficient follow-up duration for late complication analysis. Especially, the follow up duration of D group was significantly shorter than non-D group so that cardiac events or SMN results might be changed after longer follow-up. Third, there could 
be other risk factors for SMN development such as cancer predisposing genetic factors and other alkylating agents including ifosfamide. In addition, specific medical conditions which might cause cardiac events other than anthracycline could not be considered because of the limitation of retrospective design.

In conclusion, dexrazoxane had an efficacy in the prevention of lowering cardiac EFS rates in patients with higher cumulative anthracyclines. According to the multivariate analysis, the occurrence of SMN was not related to dexrazoxane. Given the efficacy of dexrazoxane as a cardioprotectant, dexrazoxane could be used in anthracycline-containing pediatric regimens.

\section{Electronic Supplementary Material}

Supplementary materials are available at Cancer Research and Treatment website (https://www.e-crt.org).

\section{Conflicts of Interest}

Conflict of interest relevant to this article was not reported.

\section{Acknowledgments}

This research was supported by a grant (12172MFDS231) from the Ministry of Food and Drug Safety, Republic of Korea.

\section{Author Details}

${ }^{1}$ Department of Pediatrics, Cancer Research Institute, Seoul National University College of Medicine, Seoul, ${ }^{2}$ Department of Pediatrics, Asan Medical Center, University of Ulsan College of Medicine, Seoul, ${ }^{3}$ Department of Pediatrics, College of Medicine, The Catholic University of Korea, Seoul, ${ }^{4}$ Department of Pediatrics, Yeungnam University Medical Center, Yeungnam University College of Medicine, Daegu, ${ }^{5}$ Department of Pediatrics, Daegu Fatima Hospital, Daegu, ${ }^{6}$ Department of Pediatrics, Korean Cancer Center Hospital, Seoul, 'Department of Pediatrics, Hanyang University Medical Center, Hanyang University College of Medicine, Seoul, ${ }^{8}$ Department of Pediatrics, Ulsan University Hospital, University of Ulsan College of Medicine, Ulsan, ' Department of Pediatrics, Chonnam National University Hwasun Hospital, Chonnam National University School of Medicine, Hwasun, ${ }^{10}$ Department of Pediatrics, Keimyung University Dongsan Medical Center, Keimyung University School of Medicine, Daegu, ${ }^{11}$ Department of Pediatrics, School of Medicine, Kyungpook National University, Daegu, ${ }^{12}$ Department of Pediatrics, Wonju Severance Christian Hospital, Yonsei University Wonju College of Medicine, Wonju, ${ }^{13}$ Department of Pediatrics, Dong-A University Hospital, Dong-A University College of Medicine, Busan, ${ }^{14}$ Department of Pediatrics, Chungbuk National University Hospital, Chungbuk National University College of Medicine, Cheongju, ${ }^{15}$ Department of Pediatrics, Kyung Hee University Medical Center, Kyung Hee University School of Medicine, Seoul, ${ }^{16}$ Department of Pediatrics, Dankook University Hospital, Dankook University College of Medicine, Cheonan, ${ }^{17}$ Department of Pediatrics, Seoul National University Bundang Hospital, Seongnam, Korea, ${ }^{18}$ Department of Pediatrics, Kagoshima University Graduate School of Medical and Dental Sciences, Kagoshima, Japan, ${ }^{19}$ Medical Research Collaborating Center, Seoul National University Hospital, Seoul, Korea

\section{References}

1. Harake D, Franco VI, Henkel JM, Miller TL, Lipshultz SE. Cardiotoxicity in childhood cancer survivors: strategies for prevention and management. Future Cardiol. 2012;8:647-70.

2. Brewster DH, Clark D, Hopkins L, Bauer J, Wild SH, Edgar $\mathrm{AB}$, et al. Subsequent hospitalisation experience of 5-year survivors of childhood, adolescent, and young adult cancer in Scotland: a population based, retrospective cohort study. Br J Cancer. 2014;110:1342-50.

3. Choi HS, Park ES, Kang HJ, Shin HY, Noh CI, Yun YS, et al. Dexrazoxane for preventing anthracycline cardiotoxicity in children with solid tumors. J Korean Med Sci. 2010;25:1336-42.

4. Barry EV, Vrooman LM, Dahlberg SE, Neuberg DS, Asselin $\mathrm{BL}$, Athale UH, et al. Absence of secondary malignant neoplasms in children with high-risk acute lymphoblastic leukemia treated with dexrazoxane. J Clin Oncol. 2008;26:
1106-11.

5. Tebbi CK, London WB, Friedman D, Villaluna D, De Alarcon PA, Constine LS, et al. Dexrazoxane-associated risk for acute myeloid leukemia / myelodysplastic syndrome and other secondary malignancies in pediatric Hodgkin's disease. J Clin Oncol. 2007;25:493-500.

6. Walker DM, Fisher BT, Seif AE, Huang YS, Torp K, Li Y, et al. Dexrazoxane use in pediatric patients with acute lymphoblastic or myeloid leukemia from 1999 and 2009: analysis of a national cohort of patients in the Pediatric Health Information Systems database. Pediatr Blood Cancer. 2013;60:616-20.

7. Vrooman LM, Neuberg DS, Stevenson KE, Asselin BL, Athale $\mathrm{UH}$, Clavell L, et al. The low incidence of secondary acute myelogenous leukaemia in children and adolescents treated with dexrazoxane for acute lymphoblastic leukaemia: a report 
from the Dana-Farber Cancer Institute ALL Consortium. Eur J Cancer. 2011;47:1373-9.

8. Seif AE, Walker DM, Li Y, Huang YS, Kavcic M, Torp K, et al. Dexrazoxane exposure and risk of secondary acute myeloid leukemia in pediatric oncology patients. Pediatr Blood Cancer. 2015;62:704-9.

9. Blanco JG, Sun CL, Landier W, Chen L, Esparza-Duran D, Leisenring W, et al. Anthracycline-related cardiomyopathy after childhood cancer: role of polymorphisms in carbonyl reductase genes: a report from the Children's Oncology Group. J Clin Oncol. 2012;30:1415-21.

10. Florescu M, Cinteza M, Vinereanu D. Chemotherapy-induced cardiotoxicity. Maedica (Buchar). 2013;8:59-67.

11. Kremer LC, van Dalen EC, Offringa M, Ottenkamp J, Voute PA. Anthracycline-induced clinical heart failure in a cohort of 607 children: long-term follow-up study. J Clin Oncol. 2001;19: 191-6.

12. Lipshultz SE, Adams MJ. Cardiotoxicity after childhood cancer: beginning with the end in mind. J Clin Oncol. 2010;28: 1276-81.

13. Lipshultz SE, Scully RE, Lipsitz SR, Sallan SE, Silverman LB, Miller TL, et al. Assessment of dexrazoxane as a cardioprotectant in doxorubicin-treated children with high-risk acute lymphoblastic leukaemia: long-term follow-up of a prospective, randomised, multicentre trial. Lancet Oncol. 2010;11:950-61.

14. Mozdzanowska D, Wozniewski M. Radiotherapy and anthracyclines: cardiovascular toxicity. Contemp Oncol (Pozn). 2015; 19:93-7.
15. Swain SM, Whaley FS, Ewer MS. Congestive heart failure in patients treated with doxorubicin: a retrospective analysis of three trials. Cancer. 2003;97:2869-79.

16. Vega-Stromberg T. Chemotherapy-induced secondary malignancies. J Infus Nurs. 2003;26:353-61.

17. Hawkins MM, Wilson LM, Stovall MA, Marsden HB, Potok $\mathrm{MH}$, Kingston JE, et al. Epipodophyllotoxins, alkylating agents, and radiation and risk of secondary leukaemia after childhood cancer. BMJ. 1992;304:951-8.

18. Haddy N, Le Deley MC, Samand A, Diallo I, Guerin S, Guibout $\mathrm{C}$, et al. Role of radiotherapy and chemotherapy in the risk of secondary leukaemia after a solid tumour in childhood. Eur J Cancer. 2006;42:2757-64.

19. Friedman DL, Whitton J, Leisenring W, Mertens AC, Hammond S, Stovall M, et al. Subsequent neoplasms in 5-year survivors of childhood cancer: the Childhood Cancer Survivor Study. J Natl Cancer Inst. 2010;102:1083-95.

20. Choi DK, Helenowski I, Hijiya N. Secondary malignancies in pediatric cancer survivors: perspectives and review of the literature. Int J Cancer. 2014;135:1764-73.

21. Bhuller KS, Zhang Y, Li D, Sehn LH, Goddard K, McBride ML, et al. Late mortality, secondary malignancy and hospitalisation in teenage and young adult survivors of Hodgkin lymphoma: report of the Childhood/Adolescent/Young Adult Cancer Survivors Research Program and the BC Cancer Agency Centre for Lymphoid Cancer. Br J Haematol. 2016; 172:757-68. 